\title{
Internação domiciliar: o perfil dos pacientes assistidos pelo Programa HU em Casa
}

\author{
I ' Daniella Reis Barbosa Martelli, ${ }^{2}$ Marília Sarmento da Silva, \\ ${ }^{3}$ Jair Almeida Carneiro, ${ }^{4}$ Paulo Rogério Ferreti Bonan, \\ ${ }^{5}$ Lais Helena Costa Rodrigues, ${ }^{6}$ Hercílio Martelli-Júnior I
}

Resumo: Internação domiciliar é uma modalidade de atendimento à saúde que está se transformando em uma alternativa importante para minimizar alguns dos principais problemas inerentes aos sistemas de saúde vigentes, especialmente os da rede pública. O objetivo do estudo foi descrever o perfil sociodemográfico e clínico da população assistida pelo Programa de Internação Domiciliar (PID) HU em Casa do Hospital Universitário Clemente de Faria da Universidade Estadual de Montes Claros. O estudo foi descritivo e retrospectivo por meio da análise de prontuários, realizada de maio de 2005 a maio de 2008. Foram analisados 137 pacientes, sendo 75 do gênero feminino $(54,7 \%)$ e 62 do masculino (45,3\%). O grupo de 61 a 80 anos foi mais prevalente $(37,2 \%)$ e $73 \%$ dos pacientes residiam em bairros periféricos do município de Montes Claros-MG. Dos agravos mais comuns na primeira internação, a pneumonia foi prevalente, 22 casos (16,1\%). A maioria dos pacientes foi encaminhada ao PID pela clinica médica $(84,7 \%)$, com intervalo de maior prevalência de duas a três internaçóes $(42,4 \%)$. Do total de pacientes, $120(87,6 \%)$ permaneceram internados por 16 a 30 dias e 51,8\% não necessitaram passar novamente pelo PID para uma segunda internação. Com relação à resolutividade clínica, 130 (94,9\%) tiveram alta clínica, no PID, na primeira internação. O PID mostrou-se ser um programa de alta resolutividade, atendendo mais ao público idoso feminino, de baixa renda e com períodos de internação relativamente curtos.

> Palavras-chave: cuidados domiciliares de Saúde; epidemiologia; SUS.

\author{
1 Pesquisadora do Centro de \\ Ciências Biológicas e da Saúde \\ da Universidade Estadual de \\ Montes Claros (Unimontes) \\ e do Hospital Universitário \\ Clemente de Faria da \\ Unimontes, Minas Gerais \\ 2 Pesquisadora, Hospital \\ Universitário Clemente de Faria \\ da Unimontes. \\ ${ }^{3}$ Pesquisador do Centro de \\ Ciências Biológicas e da Saúde \\ e do Hospital Universitário \\ Clemente de Faria da \\ Unimontes. \\ ${ }^{4}$ Pesquisador do Centro \\ de Ciências Biológicas e \\ da Saúde e do Hospital \\ Universitário Clemente de \\ Faria da Unimontes. Endereço \\ Eletrônico: pbonan@yahoo.com \\ ${ }^{5}$ Pesquisadora, Hospital \\ Universitário Clemente de Faria \\ da Unimontes. \\ ${ }^{6}$ Pesquisador do Centro de \\ Ciências Biológicas e da Saúde \\ e do Hospital Universitário \\ Clemente de Faria da \\ Unimontes. Endereço Eletrônico: \\ hmjunior2000@yahoo.com
}

Recebido em: 23/02/2010. Aprovado em: 02/12/2010. 
Nas últimas décadas, tem sido crescente, no mundo inteiro, um movimento que busca respostas para o fenômeno dos altos custos sociais com a atenção hospitalar e a insatisfatória resposta comprovada pelos indicadores de qualidade de saúde das populações (SILVA et al., 2005). Com o envelhecimento da população e a cronificação de certas doenças, modalidades alternativas à hospitalização, que utilizam o domicílio como lugar de cura/cuidado, têm apresentado rápido e expressivo crescimento no Brasil, entre elas, a internação domiciliar (MESQUITA et al., 2005).

A internação domiciliar é uma modalidade de atendimento à saúde que está se transformando em alternativa importante para minimizar alguns dos principais problemas dos sistemas de saúde, particularmente os da rede pública. $\mathrm{O}$ atendimento domiciliar, nos moldes do home care internacional, vem sendo gradativamente implementado no Brasil em diversos serviços públicos desde 1993, com o incentivo do Ministério da Saúde (MS) através do Programa de Saúde da Família (MONETTA, 2000; GARGANO et al., 2004).

O MS preconiza a internação domiciliar como uma diretriz para a equipe básica de saúde, destacando que a mesma não substitui a internação hospitalar e que deve ser sempre utilizada no intuito de humanizar e garantir maior conforto à população. Para tanto, deve ser realizada quando as condições clínicas do usuário e a situação da família o permitam (BRASIL, 1997; SILVA et al., 2005).

Assim, em decorrência da escassez de avaliações brasileiras, este estudo teve como objetivo avaliar a experiência do Programa de Internação Domiciliar (PID) HU em Casa do Hospital Universitário Clemente de Faria (HUCF) da Universidade Estadual de Montes Claros, Unimontes, Minas Gerais, Brasil.

\section{Metodologia}

O presente estudo teve caráter descritivo e retrospectivo. Avaliou-se a experiência do Programa de Internação Domiciliar (PID) HU em Casa do HUCF. Foram utilizadas informações sobre os atendimentos do PID, referentes ao período de maio de 2005 a maio de 2008. Os dados clínicos foram obtidos por meio dos prontuários dos pacientes assistidos pelo $H U$ em Casa, sendo excluídos da investigação os prontuários que estavam preenchidos incorretamente ou incompletos. As variáveis analisadas foram: número de internaçōes no $\mathrm{HU}$ em 
Casa, gênero, faixa etária, cor, estado civil, escolaridade, profissão, diagnóstico, clínica de origem, tempo e número de internações e motivo de desligamento. As avaliações dos prontuários foram sempre realizadas pelos mesmos examinadores (DRBM e JAC). Indivíduos que receberam alta na primeira internação puderam ser admitidos no PID para internaçôes subsequentes.

As informações coletadas foram transferidas para um banco de dados construído no programa SPSS $17.0^{\circledR}$ (SPSS for Windows, Chicago, EUA) e foram submetidas a análises descritivas de frequência de eventos. Este estudo foi submetido e aprovado pelo Comitê de Ética em Pesquisa da Universidade.

\section{Resultados}

Entre maio de 2005 a maio de 2008, foram analisados 137 pacientes do Programa de Internação Domiciliar HU em Casa, sendo 75 do gênero feminino (54,7\%) e 62 do masculino (45,3\%). O grupo de 61 a 80 anos foi mais prevalente (37,2\%), seguido da faixa etária acima de 81 anos (26,3\%). Quanto à escolaridade, 72 indivíduos possuíam o ensino fundamental incompleto $(52,6 \%)$. Em relação à profissão, 45 pacientes relataram exercer atividades do lar $(32,8 \%)$ e 38 eram aposentados (27,7\%). Quanto à residência, 73\% moravam em bairros periféricos do município de Montes Claros, sendo alistados 65 bairros, além do centro da cidade e da zona rural. Os dados gerais sociodemográficos são observados na Tabela 1.

Com relação aos agravos mais comuns na primeira internação, os mais prevalentes foram a pneumonia, 22 casos $(16,1 \%)$, diabetes melito, nove casos (6,6\%), tuberculose e leishmaniose visceral, sete casos cada (5,1\%). Da população assistida, 116 pacientes foram encaminhados ao $\mathrm{HU}$ em Casa pela clinica médica (84,7\%) e 10 pela clínica cirúrgica (7,3\%). Em relação ao número de internações por paciente no PID, percebeu-se que houve no mínimo uma $(51,8 \%)$ e no máximo 18 (7\%), com intervalo de maior prevalência de duas a três internaçôes $(42,4 \%)$. Quanto ao período de internação, 120 pacientes foram assistidos pelo PID por 16 a 30 dias (87,6\%). Dos 137 indivíduos que passaram pela primeira vez no $H U$ em Casa, 51,8\% não necessitaram passar novamente pelo programa para uma segunda internação e $81 \%$ para uma terceira. Com relação à resolutividade clínica, 130 pacientes tiveram alta clínica, no PID, na primeira internação (94,9\%). O tempo médio de permanência no PID mais prevalente foi de 16 a 30 dias (41,6\%). A Tabela 2 evidencia os dados referentes às variáveis de indicação, morbidades, seguimento e desfecho dos pacientes em estudo. 
Tabela 1. Variáveis Sociodemográficas (gênero, faixa etária, cor de pele, estado civil, escolaridade, profissão e bairro onde reside) relacionadas aos 137 pacientes atendidos pelo PID HU em Casa (Montes Claros, Minas Gerais, Brasil)

\begin{tabular}{|c|c|c|c|}
\hline \multicolumn{2}{|r|}{ Variáveis } & \multirow{2}{*}{$\frac{\mathbf{n}}{75}$} & \multirow{2}{*}{$\frac{\%}{54,7}$} \\
\hline Cânoro & Feminino & & \\
\hline Genero & Masculino & 62 & 45,3 \\
\hline \multirow{5}{*}{$\begin{array}{l}\text { Faixa Etária } \\
\text { (anos) }\end{array}$} & 0 a 20 & 9 & 6,6 \\
\hline & 21 a 40 & 12 & 8,8 \\
\hline & 41 a 60 & 29 & 21,2 \\
\hline & 61 a 80 & 51 & 37,2 \\
\hline & acima de 81 & 36 & 26,3 \\
\hline \multirow{4}{*}{ Cor de Pele } & Leucoderma & 29 & 21,2 \\
\hline & Melanoderma & 11 & 8,0 \\
\hline & Faioderma & 40 & 29,2 \\
\hline & Não Referido & 57 & 41,6 \\
\hline \multirow{6}{*}{ Estado Civil } & Solteiro(a) & 43 & 31,4 \\
\hline & Casado(a) & 48 & 35,0 \\
\hline & Divorciado(a) & 7 & 5,1 \\
\hline & Viúvo(a) & 36 & 26,3 \\
\hline & União estável & 1 & 0,7 \\
\hline & Não informado & 2 & 1,5 \\
\hline \multirow{8}{*}{ Escolaridade } & Sem escolaridade & 43 & 31,4 \\
\hline & Ensino Fundamental Incompleto & 72 & 52,6 \\
\hline & Ensino Fundamental Completo & 4 & 2,9 \\
\hline & Ensino Médio Incompleto & 3 & 2,2 \\
\hline & Ensino Médio Completo & 3 & 2,2 \\
\hline & Ensino Superior Incompleto & 1 & 0,7 \\
\hline & Ensino Superior Completo & 3 & 2,2 \\
\hline & Não Informada & 8 & 5,8 \\
\hline \multirow{4}{*}{ Profissão } & Aposentados & 38 & 27,7 \\
\hline & Do lar & 45 & 32,8 \\
\hline & Ativos no mercado de trabalho & 42 & 30,7 \\
\hline & Sem função específica & 12 & 8,8 \\
\hline \multirow{3}{*}{ Bairro onde reside } & Centro & 10 & 7,3 \\
\hline & Bairros adjacentes ao centro & 27 & 19,7 \\
\hline & Bairros Periféricos & 100 & 73,0 \\
\hline
\end{tabular}


Tabela 2. Variáveis relacionadas à origem do encaminhamento para a internação, à doença, à permanência e ao motivo do desligamento dos 137 pacientes atendidos pelo PID HU em Casa (Montes Claros, Minas Gerais, Brasil)

\begin{tabular}{|c|c|c|c|}
\hline & Variáveis & $\mathbf{n}$ & $\%$ \\
\hline \multirow{7}{*}{$\begin{array}{l}\text { Origem do } \\
\text { encaminhamento } \\
\text { para a internação } \\
\text { do paciente }\end{array}$} & Pronto atendimento & 3 & 2,2 \\
\hline & Clínica médica & 116 & 84,7 \\
\hline & Clínica cirúrgica & 10 & 7,3 \\
\hline & Pediatria & 2 & 1,5 \\
\hline & Não informado & 3 & 2,2 \\
\hline & Ordem judicial & 1 & 0,7 \\
\hline & Ginecologia/obstetrícia & 2 & 1,5 \\
\hline \multirow{13}{*}{ Doença de Base } & Pneumonia & 22 & 16,1 \\
\hline & Diabete Mellito & 9 & 6,6 \\
\hline & Tuberculose & 7 & 5,1 \\
\hline & Leishmaniose Visceral & 7 & 5,1 \\
\hline & Erisipela & 6 & 4,4 \\
\hline & Acidente Vascular Cerebral & 6 & 4,4 \\
\hline & Doença pulmonar obstrutiva & 4 & 2,9 \\
\hline & Úlcera em membro inferior & 4 & 2,9 \\
\hline & Insuficiência Cardíaca & 4 & 2,9 \\
\hline & Desnutrição & 3 & 2,2 \\
\hline & AIDS & 3 & 2,2 \\
\hline & Doença de Alzeheimer & 3 & 2,2 \\
\hline & Outras* & 59 & 43,1 \\
\hline \multirow{7}{*}{$\begin{array}{l}\text { Permanência total } \\
\text { do paciente no } H U \\
\text { em Casa (dias) }\end{array}$} & 15 & 14 & 10,2 \\
\hline & 16 a 30 & 57 & 41,6 \\
\hline & 31 a 45 & 14 & 10,2 \\
\hline & 46 a 60 & 29 & 21,2 \\
\hline & 61 a 75 & 6 & 4,4 \\
\hline & 76 a 90 & 10 & 7,3 \\
\hline & 91 & 7 & 5,1 \\
\hline \multirow{5}{*}{$\begin{array}{l}\text { Motivo do } \\
\text { desligamento do } \\
\text { paciente do } \\
\text { HU em Casa após } \\
\text { a } 1^{\text {a }} \text { internaçáo }\end{array}$} & Alta hospitalar & 130 & 94,9 \\
\hline & Alta a pedido & 1 & 0,7 \\
\hline & Transferência & 1 & 0,7 \\
\hline & Óbito & 4 & 2,9 \\
\hline & Não informado & 1 & 0,7 \\
\hline
\end{tabular}

* Enfermidades que somaram apenas um caso por tipo de doença. 


\section{Discussão}

O Programa de Internação Domiciliar (PID) é um modelo de internação extrahospitalar que presta serviços de assistência à saúde ao paciente que ainda necessita de cuidados especiais, mas que pode ficar internado em seu domićlio. Tem como objetivo desospitalizar o paciente, criando condiçôes familiares para que se continue o tratamento, sem perda de qualidade e de efetividade (EQUIPE..., 2004). Representa uma estratégia na reversão da atenção centralizada em hospitais e propicia a construção de nova lógica de atenção, com enfoque na promoção e prevenção à saúde e na humanização da atenção. Constitui-se como importante alternativa para os hospitais prestadores de serviço ao Sistema Único de Saúde (SUS) (EQUIPE..., 2004).

No final do século XVIII, na Europa, antes do surgimento dos grandes hospitais e da modalidade de assistência ambulatorial, os cuidados domiciliares já eram largamente empregados. Atualmente, a utilização do domicílio como espaço de atenção busca racionalizar a utilização dos leitos hospitalares e os custos da atenção, além de construir uma nova lógica de atenção centrada na vigilância à saúde e na humanização (SILVA et al., 2005).

$\mathrm{Na}$ França, a internação domiciliar é uma alternativa assistencial do setor de saúde que consiste em um modelo organizado capaz de dispensar um conjunto de cuidados e atenção, tanto em qualidade como em quantidade, para pacientes em seu domicílio, que não precisam da infraestrutura hospitalar, mas necessitam de vigilância ativa e assistência completa (NOGUEIRA et al., 2000).

$\mathrm{O}$ atendimento domiciliar brasileiro beneficia pacientes internados pelo SUS e o público alvo é a população idosa, que necessita de cuidados (EQUIPE..., 2004). O paciente e sua família contam com uma equipe multiprofissional, formada por médicos, equipe de enfermagem, fisioterapeuta, psicólogo, nutricionista e assistente social, que oferece assistência integral e contínua durante o período de Internação Domiciliar (EQUIPE..., 2004).

O documento do Ministério da Saúde sobre as diretrizes para a atenção domiciliar no SUS revela um conjunto de ações que podem ser realizadas pela internação domiciliar: procedimentos terapêuticos, educação sanitária, cuidados paliativos e visitas de monitoramento, pautando-se pelo cuidado integral, por açôes inter e transdisciplinares, devendo ser consideradas as condiçôes locais, as 
questôes habitacionais, sociais, culturais, a formação de equipes, a rede básica e a decisão do gestor local de saúde (BRASIL, 2004; LACERDA et al., 2006).

Para Lacerda (2000), a internação domiciliar é a prestação de cuidados sistematizados de forma integral e contínua no domicílio, com supervisão e ação da equipe de saúde específica, personalizada, centrada na realidade do cliente, envolvendo a família. O cliente recebe cuidados e orientaçôes sobre várias ações, pois está com o estado de saúde alterado, precisando de acompanhamento profissional. O serviço de Internação Domiciliar HU em Casa, implantado em maio de 2005, é desenvolvido por uma equipe multiprofissional do Hospital Universitário Clemente de Faria e operacionalizado exclusivamente através do SUS.

No perfil sociodemográfico dos usuários do PID HU em Casa, destacou-se a presença de indivíduos idosos do gênero feminino. Tais dados condizem com os estudos realizados por Gargano et al. (2004) e Fabrício et al. (2004). O estudo de Fabrício et al. (2004), realizado no serviço de assistência domiciliar do Hospital São Francisco, Ribeirão Preto, evidenciou a prestação de atendimento, em sua maioria, a mulheres, com idade predominantemente entre 70-80 anos. No estudo de Gargano et al (2004), a média de idade dos pacientes atendidos pelo serviço de internação domiciliar do Hospital Público de Santa Maria-RS foi de 60 anos e 50,5\% dos pacientes eram idosos. O fato de a maioria dos clientes serem idosos pode ter relação com o aumento da expectativa de vida e com a prevalência de doenças crônicas nessa faixa etária, tornando-os mais propensos à necessidade de cuidados domiciliares, principalmente pela grande dificuldade de locomoção que muitos apresentam, devido à senescência e à senilidade (FABRÍCIO et al., 2004).

Com relação aos agravos mais comuns na primeira internação dos usuários do PID HU em Casa, os mais prevalentes foram a pneumonia, 22 casos (16,1\%), diabetes melito, nove casos (6,6\%), tuberculose e leishmaniose visceral, sete casos cada (5,1\%). Os diagnósticos prevalentes no estudo de Fabrício et al. (2004) foram doença neurológica (27,0\%), neoplásica (17,0\%), osteomuscular (12,0\%) e cardiovascular (12,0\%). Entre os motivos de internação no estudo realizado por Gargano et al. (2004), as principais doenças foram infecção respiratória (35,5\%), complicações de neoplasia (12,0\%), complicações de acidente vascular cerebral $(11,0 \%)$, cardiopatias $(9,0 \%)$, infecçōes urinárias $(9,0 \%)$ e infecções cutâneas $(6,5 \%)$. Tais dados demonstram certa diferença quanto aos principais diagnósticos dos pacientes assistidos pela internação domiciliar nesses serviços. 
Talvez as condições encontradas estejam relacionadas ao perfil epidemiológico típico de cada região, revelando os agravos mais comuns em que a população idosa possa estar mais vulnerável.

Quanto ao número de internações no PID HU em Casa, houve prevalência de duas a três internaçôes $(42,4 \%)$, sendo que 120 pacientes foram assistidos por um período de 16 a 30 dias (87,6\%). Dos 137 indivíduos que passaram pela primeira vez no $H U$ em Casa, 51,8\% não necessitaram passar novamente pelo programa para uma segunda internação. Com relação à resolutividade clínica, 130 pacientes tiveram alta do PID na primeira internação $(94,9 \%)$ e quatro foram a óbito (2,9\%). Gargano et al. (2004) afirmaram que o tempo médio de permanência dos pacientes no programa em seu estudo foi aproximadamente 20 dias. Quanto à evolução clínica observada, 59\% dos pacientes receberam alta com melhora do quadro, $28 \%$ faleceram, $7 \%$ tiveram seu quadro clínico inalterado e $6 \%$ foram encaminhados a outras instituições.

Cotta et al. (2001) destacam que a internação domiciliar deve ter caráter transitório e os dados encontrados o corroboram, visto que o tempo médio de permanência dos pacientes no PID dos dois estudos analisados foi praticamente equivalente, em torno de 20 dias, e a maioria recebeu alta com melhora do quadro apresentado. Todavia, destaca-se a diferença entre a porcentagem de óbito encontrada entre os dois estudos. Provavelmente, o maior índice evidenciado por Gargano et al. (2004) esteja relacionado às características mais agressivas das doenças que a população de seu estudo foi acometida. Neste sentido, a internação domiciliar é uma modalidade que tem se revelado uma opção segura e eficaz, direcionada a pacientes portadores de doenças crônicas ou agudas (FREITAS et al., 2000; LACERDA et al., 2006). Está adquirindo cada vez mais importância nas distintas organizações sanitárias e sociais, acompanhada por estudos que preveem um aumento dos serviços de atendimento domiciliar, assinalando que diferentes setores a consideram como alternativa viável e promissora (LACERDA, 1999; LACERDA et al., 2006).

Fabrício et al. (2004) destacaram que prestar assistência domiciliar não é apenas concretizar uma nova modalidade de assistência à saúde, mas possibilitar que as pessoas experimentem uma nova forma de atenção à saúde, aliada a conhecimento e tecnologia. É realizar assistência baseada na realidade de cada indivíduo, proporcionando cuidado individualizado e mais humanizado. Com 
isto, percebe-se que a internação domiciliar visa a propiciar uma recuperação

mais rápida do paciente, que fica junto de seus familiares. Diminui a incidência de infecções hospitalares, permitindo um tratamento específico de sua doença e, finalmente, libera leitos nos hospitais para pacientes que requeiram tratamento mais complexo (EQUIPE..., 2004). Para Duarte e Diogo (2000), a expansão do sistema de saúde gerou um custo muito alto no sistema público e suplementar. A assistência domiciliar veio atender, dentre outras, também essa demanda, uma vez que tende a reduzir os custos assistenciais, comparando-se às mesmas intervenções realizadas em ambiente hospitalar (DUARTE; DIOGO, 2000).

A internação domiciliar, portanto, tem como objetivos acelerar o processo de recuperação através da proximidade do ambiente familiar; reduzir custos com internações e reinternações; diminuir os riscos de infecçôes hospitalares; proporcionar um ambiente mais humano ao paciente; diminuir a incidência de depressão por causa da doença; aumentar a rotatividade dos leitos; liberar leitos para pacientes críticos; reintegrar o paciente à vida normal; integrar médicofamília-paciente, proporcionando melhores condições psicológicas ao doente (EQUIPE..., 2004).

\section{Referências}

BRASIL. Ministério da Saúde. Diretrizes para a atenção domiciliar no sistema único de saúde. Brasília: MS, 2004.

BRASIL. Portaria no 1892, de 18 de dezembro de 1997. Dispõe sobre a internação domiciliar no SUS e dá outras providências. Brasília (DF): Diário Oficial da República Federativa do Brasil; 22 dez 1997.

COTTA, R.M.M. et al. La hospitalización domiciliaria: antecedentes, situación actual y perspectivas. Revista Panamericana de Salud Pública, v. 10, n. 1, p. 45-55, jul. 2001.

DUARTE, Y.A.O.; DIOGO, M.J.E. Atendimento domiciliar: um enfoque gerontológico. São Paulo: Atheneu, 2000.

EQUIPE DE INTERNAÇÃO Domiciliar do Hospital Dom João Becker. Internação domiciliar. Boletim da Saúde, v. 18, n. 2, 2004.

FABRÍCIO, S.C.C. et al. Assistência domiciliar: a experiência de um hospital do interior paulista. Rev Latino-Am Enfermagem, v. 12, n. 5, p.721-6, 2004.

FREITAS, A.V.S.; BITTENCOURT, C.M.M.; TAVARES, J.L. Atuação da enfermagem no serviço de internação domiciliar: relato de experiência. Revista Baiana de Enfermagem, v. 13, n. 1/2, p. 103-107, 2000. 
GARGANO, F. et al. Internação domiciliária: uma experiência no sul do Brasil. Revista $A M R I G S$, v. 48, n. 2, p. 90-94, 2004.

LACERDA, M.R. Tornando-se profissional no contexto domiciliar: vivência do cuidado da enfermeira. 2000. Tese (Doutorado em Filosofia da Enfermagem) - Universidade Federal de Santa Catarina, Florianópolis, 2000.

LACERDA, M.R. Internação domiciliar. In: CONGRESSO BRASILEIRO DE ENFERMAGEM, 51., 1999, Florianópolis. Anais... Florianópolis: ABEn, 1999.

LACERDA, M.R. et al. Atenção à Saúde no Domicílio: modalidades que fundamentam sua prática. Saúde e Sociedade, v. 15, n. 2, p. 88-95, 2006.

MESQUITA, S.R.A.M. et al. Programa Interdisciplinar de Internação Domiciliar de Marília-SP: custos de materiais consumidos. Rev. Latino-am Enfermagem, v. 13, n. 4, p. 555-61, 2005.

MONETTA, L. A. Especialidade no atendimento domiciliário. São Paulo: Atheneu, 2000.

NOGUEIRA, J.M.S. et al. Enfermos crônicos domiciliares: valorização integral físico-cognitiva e caídas durante 3 anos de seguimento. Atención Primária, v. 25, n. 3, p. 16-17, 2000.

SILVA, K.L. et al. Internação domiciliar no Sistema Único de Saúde. Rev. Saúde Pública, v. 39, n. 3, p. 391-7, 2005. 


\section{Abstract}

Home care: profile of patients attended by a home care program

The home care is a modality of health care which is becoming an important alternative to minimize some of mainly relevant problems of world health, especially the public health network. This paper aimed to describe the socio-demographic and clinical population assisted by the Home Care Program HU em Casa, of the University Hospital Clemente de Faria, Universidade Estadual de Montes Claros. It is a descriptive and retrospective study analyzing medical records from May 2005 to May 2008.

Of the 137 analyzed patients, 75 were females $(54.7 \%)$ and 62 males (45.3\%). Concerning age, the 61-80 year group was the most prevalent (37.2\%) and $73 \%$ lived and was attended in the peripheral districts of Montes Claros city. Among the most important diseases in the first admission, the most prevalent was pneumonia (22 cases, $16.1 \%$ ). Most patients were referred to the HU em Casa Home Care Program for the medical clinic (84.7\%) with a range of higher prevalence of 2 to 3 admissions (42.4\%). Of the total patients, $120(87.6 \%)$ stayed in hospital for 16 to 30 days. Referring to resolutivity, 130 (94.9\%) patients were discharged to the program on the first admission. The PID proved to be a high-solving program, attended mainly elderly women with low income and with periods of relatively short hospitalization.

Key words: home care; epidemiology; SUS. 